\title{
Effect of Number Family Instruction on Improving Fluency of Addition and Subtraction Facts in Japanese Second-Grade Students
}

\author{
Wataru Noda' ${ }^{1}$, Junko Tanaka-Matsumi ${ }^{2}$ \\ ${ }^{1}$ Faculty of Education, Osaka Kyoiku University, Osaka, Japan \\ ${ }^{2}$ Department of Integrated Psychological Science, Kwansei Gakuin University, Nishinomiya, Japan \\ Email: wnoda@cc.osaka-kyoiku.ac.jp
}

How to cite this paper: Noda, W., \& Tanaka-Matsumi, J. (2017). Effect of Number Family Instruction on Improving Fluency of Addition and Subtraction Facts in Japanese Second-Grade Students. Psychology, 8, 2377-2389.

https://doi.org/10.4236/psych.2017.814149

Received: November 4, 2017

Accepted: December 5, 2017

Published: December 8, 2017

Copyright $\odot 2017$ by authors and Scientific Research Publishing Inc. This work is licensed under the Creative Commons Attribution International License (CC BY 4.0).

http://creativecommons.org/licenses/by/4.0/

\begin{abstract}
The present study examined the effect of number family instruction on improving fluency of addition and subtraction facts through the "cover-copycompare" and a timed trial procedure. Three second-grade students in Japanese public elementary school participated in this study. We used a multipleprobe design across two sets of materials for each student. Throughout the study, we measured the number of correct and incorrect digits in 30 second assessments of addition and subtraction facts and in 30 second assessments of missing numbers. Intervention consisted of teaching the concept of a number family, "cover-copy-compare" for number families, and using reward contingency for beating one's previous score in 30 second timed trials of missing numbers. The results showed that students improved their fluency of addition and subtraction facts. We discussed the utility of number family instruction to improve fluency of addition and subtraction facts with regrouping.
\end{abstract}

\section{Keywords}

Number Family, Cover-Copy-Compare, Fluency, Addition, Subtraction

\section{Introduction}

Supporting students with academic difficulties as they master basic academic skills is an important agenda in elementary school education. Practicing basic academic skills until students reach fluency can contribute to the maintenance and generalization of the acquired skills and to the learning of more complex skills (Binder, 1996; Johnson \& Street, 2004). Lin and Kubina (2005) showed that fluency of multiplication facts correlated positively with fluency of complex 
multiplication problems. Noda (2011) reported that fluency of math facts predicted mathematics achievement in Japanese elementary school students. These findings suggest the importance of fluency training in addition to basic accuracy training in math instruction.

Behavioral research has demonstrated the effectiveness of fluency-based instruction to improve calculating, especially when solving math facts (e.g., Johnson \& Street, 2004; Skinner, McLaughlin, \& Logan, 1997; Stein, Kinder, Silvert, \& Carnine, 2006). Teaching number families is one of the behavioral instructional methods for improving accuracy and fluency in basic math facts (e.g., Johnson \& Street, 2004; Stein et al., 2006). Number family refers to a set of three numbers from which we can create four basic math facts. For example, we can create four basic math facts $(3+4=7,4+3=7,7-3=4$, and $7-4=3)$ from the number family 3,4 , and 7 .

Math instruction based on number family has three advantages. First, students can learn four math facts through reciting only one number family (a set of three numbers). Second, instruction based on number families will help the students solve math facts without using finger counting strategies and without depending on prompts (e.g., pencils, circles). Many students with academic difficulties cannot recall math facts without resorting to counting strategies (e.g., counting fingers, pencils) when they solve addition and subtraction facts (e.g., Gersten \& Chard, 1999; Hasselbring, Goin, \& Bransford, 1988). Third, number family instruction has educational utility for students with academic difficulties. Students with academic difficulties use counting strategies with concrete objects (e.g., fingers, pencils, and written circles) when they solve addition and subtraction facts (Gersten \& Chard, 1999). Therefore, if the math facts include a number that exceeds 10 , the difficulty of solving the math facts increases rapidly, and quite a few students engage in off-task behavior or disruptive behavior. Students' off-task behaviors increase especially when they are taught addition and subtraction with regrouping.

Both Direct Instruction (Stein et al., 2006) and the Morningside Model of Generative Instruction (Johnson \& Street, 2004) use number family instruction to teach math facts. Although these behavioral instruction models demonstrated their effectiveness using standardized achievement tests, few studies examined the effectiveness of number family instruction itself. In the present study, we evaluated the effect of number family instruction on the improvement of addition and subtraction facts with regrouping in a Japanese elementary school. Computation is one of the most difficult skills for students with academic difficulties in Japan to master (Japanese Ministry of Education, Culture, Sports, Science \& Technology, 2003).

To teach number families, we used the "cover-copy-compare" ("CCC") procedure based on Skinner, McLaughlin, and Logan (1997). CCC is a self-managed intervention that provides a series of learning trials within a short period of time. Several studies showed the effects of CCC on learning spelling (Hansen, 1978), geography (Skinner, Belfiore, \& Pierce, 1992) and math (e.g., Codding, Eckert, 
Fanning, Shiyo, \& Solomon, 2007; Skinner, Turco, Beatty, \& Rasavage, 1989). In the current study, we also used a timed trial procedure (e.g., Rhymer, Skinner, Henington, D’Reaux, \& Sims, 1998; Van Houten \& Thompson, 1976) to achieve fluency of reciting number families. The purpose of the present study was to investigate whether teaching number families through the CCC and a timed trial procedure would improve fluency of addition and subtraction with regrouping.

\section{Method}

\subsection{Participants}

Three second-grade students (YOSHI, TAKU, and SHU) in a Japanese public elementary school regular classroom participated in this study. We chose these second-grade students because the difficulty of mathematics contents for third-grade increases in Japanese school education. Students are expected to acquire addition and subtraction skills by the end of the second-grade in Japan. Second-grade students are therefore good candidates for remedial math instructions. YOSHI and TAKU used finger counting or drew circles to count while they were solving addition and subtraction facts with regrouping. They needed tremendous effort to solve these problems. After they finally solved a few problems, their off-task behavior (e.g., getting out of their seats and putting their heads down on the desks) increased. Apparently, repeated practice with counting strategies alone was not effective to improve their fluency of addition and subtraction facts. We included SHU, who did not have academic or behavioral problems, to see how he would respond to the experimental instruction. According to his homeroom teacher's report, SHU had no academic or behavioral difficulties. We administered a standardized mathematics achievement test (Tatsuno et al., 2009) to assess the overall achievement level. This test adopts a standard score to evaluate students' academic achievement with the mean standard score of 50 and a standard deviation of 10 . Standard scores of achievement tests for mathematics were 36 for YOSHI, 31 for TAKU and 52 for SHU.

We conducted the present study in February and March, which were the last two months of the academic year in Japan. The first author, serving as an instructor, conducted instructional sessions in a classroom for approximately 45 minutes in the form of an afterschool small group session for the three students. Instructional sessions were conducted twice per week. Parents agreed for their children to participate in after school session and the school principal and homeroom teachers gave their informed consent for the three students to participate in this study.

\subsection{Target Behaviors and Dependent Variables}

Target behaviors of this study were reciting number families and solving addition and subtraction facts with regrouping. Reciting number families was defined as filling in the blanks in the missing number tasks (e.g., 5, 6, _). We counted the number of correct and incorrect digits in the missing number tasks 
during 30 second timed trials. Solving addition and subtraction facts with regrouping was defined as seeing the problems and then writing the answers. We measured the number of correct and incorrect digits in 30 second timed trials.

\subsection{Experimental Design}

We used a multiple-probe design across two materials (Barlow \& Hersen, 1984) to examine the effects of the intervention. We began the intervention in a sequential fashion for two materials following baseline. During the intervention for material $\mathrm{A}$, we conducted the probes for material $\mathrm{B}$ to confirm the experimental control of the effect of the intervention. In probe sessions for material B, we gave no feedback about their performance on material B except for praising their on-task behaviors. We recorded the number of correct and incorrect digits of both materials during baseline and intervention. To assess the maintenance of the intervention effect, we recorded the number of correct and incorrect digits of material A after completing the intervention for material A.

\subsection{Materials}

We selected two sets of number families. Considering the ease of learning for students, we used material A including number families starting with 5 and material B including number families starting with 6 . Table 1 shows the number families and computation problems of each set of materials. Each set of materials included three number families and 12 addition and subtraction facts with regrouping.

We developed assessment worksheets that included 40 addition and subtraction problems with regrouping to gather assessment data. Worksheets of material A and material B included 12 types of problems, respectively. The orders of problems were randomized. For the missing number task, we made a worksheet that included 40 problems of missing number tasks (e.g., 5, 6, _). The position of blank spaces and the order of each number in each problem were randomized.

We created the worksheet with three columns for the CCC procedure (Figure 1). There are three number families in the left columns. Each number family appeared three times in randomized order. In the central columns, three squares were provided for check marking. There are three squares for writing down each number from the number families in the right columns.

Table 1. Materials used in this study.

\begin{tabular}{|c|c|c|}
\hline & Number Family & Addition \& Subtraction Facts \\
\hline \multirow{3}{*}{ Material A } & $5,6,11$ & $5+6=11,6+5=11,11-5=6,11-6=5$ \\
\hline & $5,7,12$ & $5+7=12,7+5=12,12-5=7,12-7=5$ \\
\hline & $5,8,13$ & $5+8=13,8+5=13,13-5=8,13-8=5$ \\
\hline \multirow{3}{*}{ Material B } & $6,7,13$ & $6+7=13,7+6=13,13-6=7,13-7=6$ \\
\hline & $6,8,14$ & $6+8=14,8+6=14,14-6=8,14-8=6$ \\
\hline & $6,9,15$ & $6+9=15,9+6=15,15-6=9,15-9=6$ \\
\hline
\end{tabular}




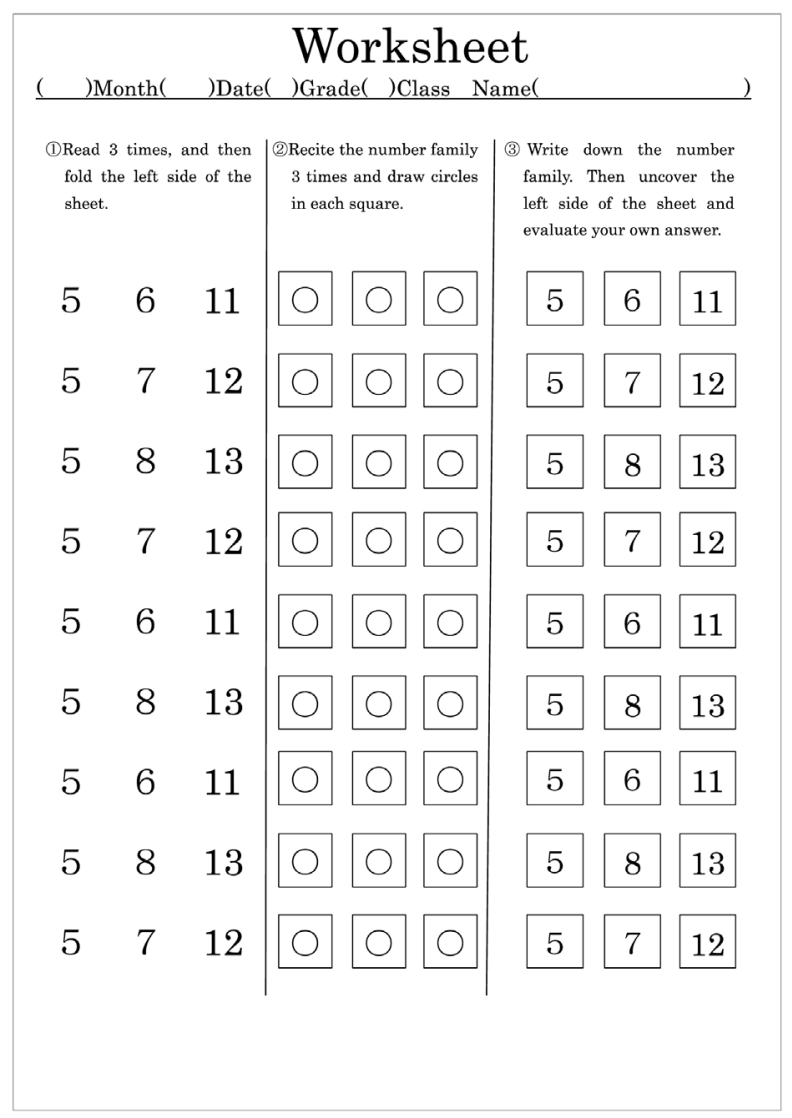

Figure 1. A sample worksheet used in the "cover-copycompare" procedure.

We used feedback sheets to deliver graphical feedback of performance of the missing number tasks for each student. In addition, we used stickers that the students preferred to reinforce accurate and fluent responses as well as on-task behavior.

\subsection{Procedures}

Baseline. Thirty second timed trials of each material (A and B) were conducted. We defined a trial as one 30 second timed trial. The instructor did not give feedback about the students' performance except for praising their on-task behavior. On-task behavior was defined as listening to the instructor's direction and engaging in the assigned tasks (timed trial, CCC, and missing number task).

Intervention. In the first session of the intervention, the instructor explained number families to the students ("we can create four math facts from the three numbers of a number family") and then practiced creating four math facts from a number family based on the instructional protocols of Stein et al. (2006). The instructor briefly reviewed how to create math facts from one number family at the start of each session. After the second session, CCC and the missing number task for number families were conducted twice per session. Intervention proceeded in the following order on and after the second session: 30 second timed 
trials for addition and subtraction facts, CCC, missing number task, CCC, missing number task, and 30 second timed trials for addition and subtraction facts. We conducted timed trials for addition and subtraction facts twice per session during intervention and used each student's responses as the assessment data.

At the beginning and end of the intervention session, the students solved addition and subtraction facts in writing as fast as possible in 30 second timed trials, and the instructor gave no feedback about the performance. The instructor only praised the students' on-task behavior. We used a CCC procedure to establish the accuracy of reciting number families. The CCC procedure in this study was composed of six steps: 1 ) reading the number family three times, 2) folding the left side of the practice sheet and covering the number family, 3) reciting the number family three times, 4) writing down the number family on the right side of the sheet, 5) uncovering the number family, and 6) evaluating their own responses. In the CCC procedure in this study, the students practiced number families in choral responding format in the classroom. The instructor orally cued the timing of each of the six CCC steps. After the CCC, the students practiced the missing number task to improve their fluency of reciting number families. In the missing number task, students filled in the blanks of number families in 30 second timed trials. If students beat their highest previous score, the instructor gave verbal praise and the student's preferred stickers. In the last part of each session, the instructor gave all students the stickers they preferred to reward their participation in the session.

Post test. After the completion of the intervention for material A, 30 second timed trials for material A were conducted once per session to gather assessment data. The instructor gave no feedback to the students except for praising the students' on-task behavior.

\subsection{Interscorer Agreements and Procedural Integrity}

The first author and a graduate student who was not informed the purpose of this study scored the students' performance. To compute interscorer agreement, the graduate student randomly selected $37 \%$ of the worksheets (12 out of 33) for YOSHI, $41 \%$ of the worksheets (14 out of 33 ) for TAKU, and $43 \%$ of the worksheets (13 out of 31) for SHU and scored them independently. The percentages of interscorer agreements on correct and incorrect digits were calculated by dividing the number of agreements by the number of agreements plus disagreements and multiplying by 100 . Interscorer agreements for the three students were all $100 \%$.

We made a manual of all intervention procedures, and the instructor used it to follow the intervention protocol during intervention phase. The instructor checked each component during intervention. The instructor himself assessed procedural integrity using an intervention manual. Procedural integrity was calculated on all sessions by dividing the total number of procedures by the number of procedures recorded as conducted correctly by the instructor. Mean 
procedural integrity was $100 \%$.

\section{Results}

\subsection{Missing Number Task}

YOSHI and SHU demonstrated high accuracy (more than 87.5\%) for missing number tasks throughout the study and improved fluency for missing number tasks. The number of correct digits for YOSHI increased from 8 to 13 for material A and from 8 to 12 for material B. SHU improved his fluency of the missing number task from 13 to 21 for material $\mathrm{A}$ and from 9 to 23 for material $\mathrm{B}$. The percent of correct digits from TAKU were $50 \%$ for material A and $0 \%$ for material B at each first trial. Ultimately, TAKU improved accuracy up to $90 \%$ for material A and $100 \%$ for material B at each last trial. In addition, the numbers of correct digits for TAKU increased from the lowest score of 4 to the higher score of 12 for material $\mathrm{A}$, and from the lowest of 0 to 12 for material $\mathrm{B}$.

\subsection{Addition and Subtraction Facts}

Figures 2-4 show the numbers of correct and incorrect digits for each student in the 30 second timed-trials of addition and subtraction facts. In each figure, the upper panel shows the result of material $\mathrm{A}$, and the bottom panel shows the result of material B. The horizontal axis indicates the number of trials, and the vertical axis indicates the number of correct (filled circle) and incorrect (blank circle) digits for both material A and material B to enable performance comparisons between experimental and probe sessions.

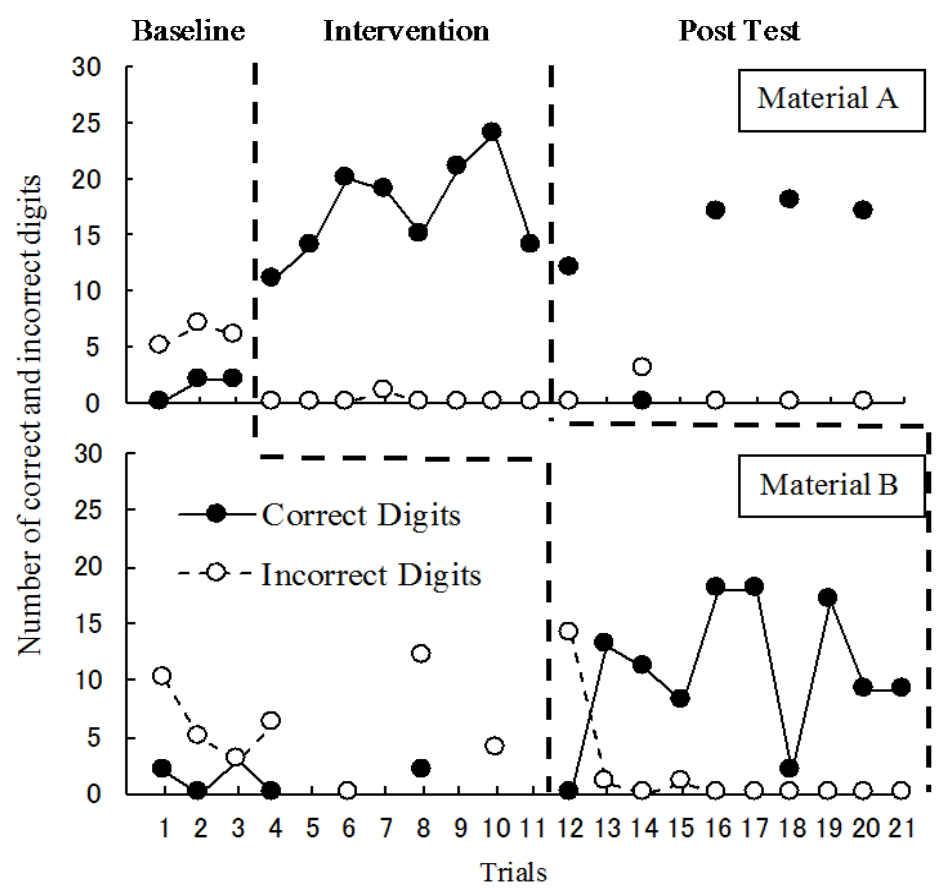

Figure 2. Number of correct and incorrect digits for YOSHI in 30 second timed trials of addition and subtraction facts. 


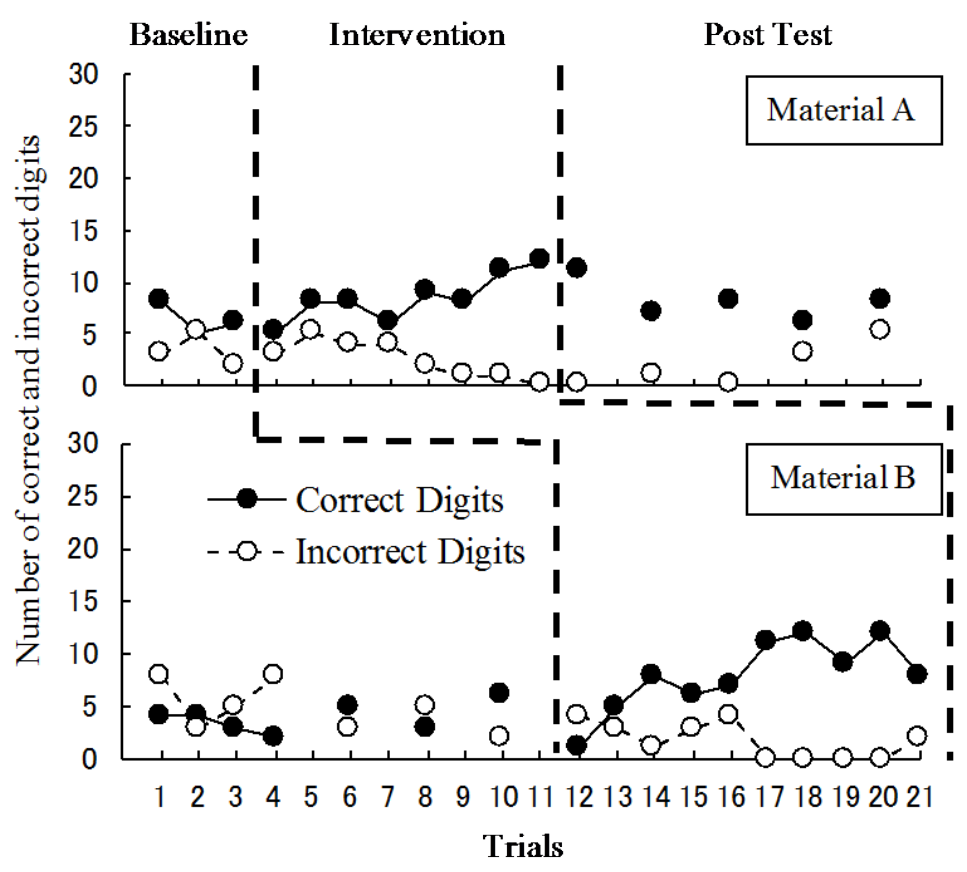

Figure 3. Number of correct and incorrect digits for TAKU in 30 second timed trials of addition and subtraction facts.

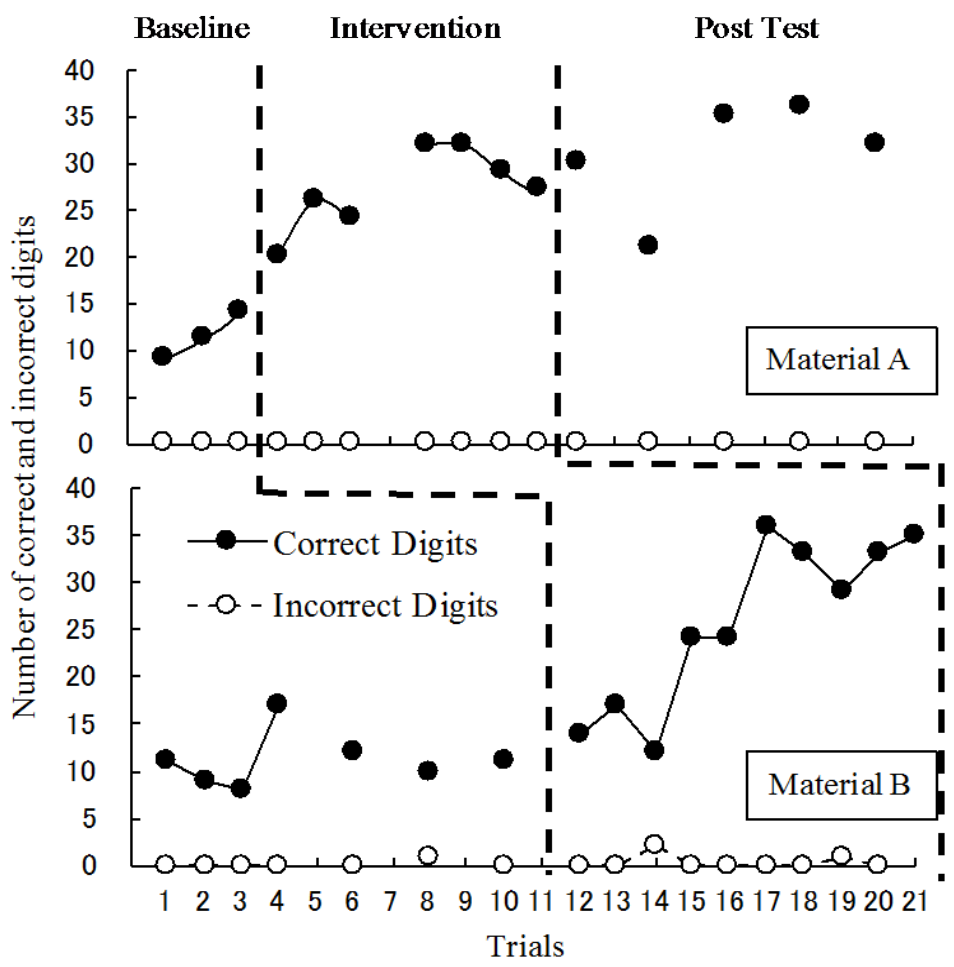

Figure 4. Number of correct and incorrect digits for SHU in 30 second timed trials of addition and subtraction facts.

When we introduced the intervention for material A, all three students' number of correct digits increased and incorrect digits decreased for this material. 
On the other hand, no clear change was found in the number of correct and incorrect digits for material B for all three students during intervention for material A. Although the number of correct digits decreased slightly for YOSHI and TAKU, and the number of incorrect digits for TAKU increased slightly in the last two probes, the intervention effect for material A was maintained through post tests for all three students. When we introduced the intervention for material B, all students improved their number of correct and incorrect digits as shown in the bottom half of each figure for each student.

To examine the intervention effect through statistical analysis, we calculated the Tau-U (Parker, Vannest, Davis, \& Sauber, 2011). Tau-U is a method for measuring data non-overlap between two phases (baseline vs. intervention). Tau-U is distribution free, and controls positive baseline trend (Parker et al., 2011). Table 2 shows the result of Tau-U for comparing the two phases for

Table 2. Analysis of the intervention effect based on Tau-U.

\begin{tabular}{|c|c|c|c|c|c|c|}
\hline & & $S$ & $T A U$ & $V A R s$ & $S D$ & $Z$ \\
\hline \multirow{8}{*}{ YOSHI } & Material A & & & & & \\
\hline & $\mathrm{BL}$ trend & 2 & .67 & 3.67 & 1.91 & $1.04^{\text {n.s. }}$ \\
\hline & IV trend & 11 & .39 & 65.33 & 8.08 & $1.36^{\text {n.s. }}$ \\
\hline & BL vs IV & 22 & .92 & 96 & 9.80 & $2.25^{\star}$ \\
\hline & Material B & & & & & \\
\hline & $\mathrm{BL}$ trend & 5 & .24 & 44.33 & 6.66 & $.75^{\text {n.s. }}$ \\
\hline & IV trend & 3 & .07 & 125 & 11.18 & $.27^{\text {n.s. }}$ \\
\hline & BL vs IV ${ }^{\mathrm{a}}$ & 48 & .69 & 420 & 20.49 & $2.34^{*}$ \\
\hline \multirow{8}{*}{ TAKU } & Material A & & & & & \\
\hline & $\mathrm{BL}$ trend & -1 & -.33 & 3.67 & 1.91 & $-.52^{\mathrm{n} . s}$ \\
\hline & IV trend & 19 & .68 & 65.33 & 8.08 & $2.35^{\star}$ \\
\hline & BL vs IV ${ }^{a}$ & 14 & .58 & 96 & 9.80 & $1.43^{\text {n.s. }}$ \\
\hline & Material B & & & & & \\
\hline & $\mathrm{BL}$ trend & 3 & .14 & 44.33 & 6.66 & $.46^{\text {n.s. }}$ \\
\hline & IV trend & 27 & .60 & 125 & 11.18 & $2.42^{*}$ \\
\hline & BL vs IV ${ }^{\mathrm{a}}$ & 49 & .70 & 420 & 20.49 & $2.39^{*}$ \\
\hline \multirow{8}{*}{ SHU } & Material A & & & & & \\
\hline & $\mathrm{BL}$ trend & 3 & 1 & 3.67 & 1.91 & $1.57^{\text {n.s. }}$ \\
\hline & IV trend & 8 & .38 & 44.33 & 6.66 & $1.20^{\text {n.s. }}$ \\
\hline & BL vs IV ${ }^{a}$ & 18 & .86 & 77 & 8.78 & $2.05^{\star}$ \\
\hline & Material B & & & & & \\
\hline & $\mathrm{BL}$ trend & 2 & .10 & 44.33 & 6.66 & $.30^{\text {n.s. }}$ \\
\hline & IV trend & 29 & .64 & 125 & 11.18 & $2.59^{\star}$ \\
\hline & $\mathrm{BL}$ vs $\mathrm{IV}^{\mathrm{a}}$ & 62 & .89 & 420 & 20.49 & $3.03^{*}$ \\
\hline
\end{tabular}

${ }^{a}$ controlled for positive baseline trend, $\mathrm{BL}=$ baseline, $\mathrm{IV}=$ Intervention, ${ }^{*} \mathrm{p}<.05$. 
material A and material B separately. Differences in all comparisons, except for material A in TAKU, were statistically significant $(p s<.05)$.

\section{Discussion}

The purpose of the present study was to investigate whether teaching number families through the CCC and a timed-trial procedure would improve fluency of addition and subtraction with regrouping. The results revealed that all three students improved their fluency of addition and subtraction facts when teaching of number families was introduced. We used a multiple-probe design across two materials and confirmed the experimental control for all three students. Teaching number families proved to be an effective method to improve fluency of addition and subtraction facts for students. Previous large-scale studies of behavioral instruction model (e.g., Direct Instruction, Morningside model of generative instruction) demonstrated the effects of number family instruction as part of more comprehensive programs. The present study demonstrated an independent effect of number family instruction tailored to each individual student in Japan.

In Japanese elementary schools, teachers typically teach students counting strategies with concrete objects (e.g., fingers, pencils, and written circles) when they teach math facts. Most students who depend on counting strategies tend to exhibit difficulty in solving addition and subtraction problems that use regrouping. YOSHI and TAKU used counting strategies to solve math facts and they showed very low accuracy in solving addition and subtraction with regrouping before the intervention. However, YOSHI rapidly improved his accuracy and fluency, and TAKU gradually improved his accuracy and fluency during the intervention. They improved their accuracy and fluency of addition and subtraction facts with regrouping in a relatively short period of time (five $45 \mathrm{~min}$ sessions), and they hardly used counting strategy after the intervention was introduced. These results suggest that a number family component in the intervention procedure led to their independence from counting strategies.

Combining the CCC and a timed-trial procedure with graphical feedback and stickers as reinforcers was effective when students learned the number families. CCC gives students many opportunities to respond with relatively fewer error responses and immediate feedback, and timed-trials with graphical feedback provide a clear track of their improvement. Incorporating these two procedures into teaching number families is an effective and efficient instructional method to improve the academic performance of students with academic difficulties. In addition, fluency of addition and subtraction facts for SHU increased rapidly through participation in the intervention. SHU did not have academic difficulties and showed high accuracy in solving addition and subtraction facts with regrouping before the intervention. Although further research is needed, the results of the present study suggest that all students with or without academic difficulties may benefit from the intervention procedures used in this study. Awaiting further systematic research, the current instructional strategies may be 
applied to general classroom instructions for all students.

At baseline, YOSHI and TAKU showed off-task behavior when the instructor was to start the timed-trials (e.g., taking a long time to sit down, saying "I want to try another task"). However, their off-task behavior decreased during the intervention. Although we could not gather observational data for off-task behavior, intervention procedures in the present study may be effective for students who exhibit off-task behavior to escape or avoid a difficult task such as addition and subtraction with regrouping as in this study.

Although the present study provides evidence for the effectiveness of number family instruction on the improvement of fluency of basic math facts through the CCC and a timed-trial procedure, we need further research on some important points. First, we need to assess the long-term maintenance of the intervention effect. This study assessed maintenance for only two weeks for only material A as post tests in each student. In addition, although the intervention effect was maintained for YOSHI and SHU, the number of incorrect digits for TAKU slightly increased during post tests. We need further research to identify the variables leading to better maintenance for TAKU. Second, we should investigate the generalization of the intervention effect. We would ideally test whether or not students acquired addition and subtraction facts by using a new set of math facts that did not appear in Materials A and B. Third, social validity such as acceptability of the intervention needs to be assessed. In another study, we assessed the acceptability of an intervention using CCC and timed-trials to improve fluency of multiplication facts and the teacher's/students' acceptance of the intervention proved to be excellent (Noda \& Tanaka-Matsumi, 2014). Few teachers use number families to teach math facts in Japanese education. We need data on the acceptability of the intervention that include number families. Finally, further research is needed to investigate effective means of incorporating intervention procedures that include number families, CCC, and timed-trials into the regular classroom instructional environment.

\section{Conclusion}

In conclusion, this study demonstrated that teaching number families effectively and efficiently improved fluency of addition and subtraction facts in elementary school students. This study showed independent effects of specifically teaching number families and addition and subtraction facts using the adapted CCC procedure in Japan. The results provide additional support for evidence-based educational programming in basic mathematics. Teaching number families may contribute to the effective remediation of computation skills in students with academic difficulties.

\section{References}

Barlow, D. H., \& Hersen, M. (Eds.) (1984). Single-Case Experimental Designs: Strategies for Studying Behavior Change (2nd ed.). Oxford: Pergamon Press.

Binder, C. (1996). Behavioral Fluency: Evolution of a New Paradigm. The Behavior Ana- 
lyst, 19, 163-197. https://doi.org/10.1007/BF03393163

Codding, R. S., Eckert, T. L., Fanning, E., Shiyko, M., \& Solomon, E. (2007). Comparing Mathematics Interventions: The Effects of Cover-Copy-Compare Alone and Combined with Performance Feedback on Digits Correct and Incorrect. Journal of Behavioral Education, 16, 125-141. https://doi.org/10.1007/s10864-006-9006-x

Gersten, R., \& Chard, D. (1999). Number Sense: Rethinking Arithmetic Instruction for Students with Mathematical Disabilities. The Journal of Special Education, 33, 18-28. https://doi.org/10.1177/002246699903300102

Hansen, C. L. (1978). Writing Skills. In N. G. Haring, T. C. Lovitt, M. D. Eaton, \& C. L. Hansen (Eds.), The Fourth R: Research in the Classroom (pp. 93-126). Columbus, OH: Merrill.

Hasselbring, T. S., Goin L., \& Bransford, J. D. (1988). Developing Math Automaticity in Learning Handicapped Children: The Role of Computerized Drill and Practice. Focus on Exceptional Children, 20, 1-7.

Johnson, K. R., \& Street, E. M. (2004). The Morningside Model of Generative Instruction: What It Means to Leave No Child Behind. Concord, MA: Cambridge Center for Behavioral Studies. https://doi.org/10.1016/B978-012506041-7/50015-2

Lin, F., \& Kubina, R. M. (2005). A Preliminary Investigation of the Relationship between Fluency and Application for Multiplication. Journal of Behavioral Education, 14, 73-87. https://doi.org/10.1007/s10864-005-2703-Z

Ministry of Education, Culture, Sports, Science \& Technology (2003). Kongo no tokubetsushienkyoiku no arikata nit suite (Saisyuhokoku). [Prospect of Special Needs Education in the Future (Final Report)]. Tokubetsushienkyoiku no arikatanikansurutyosakenkyukyoryokusyakaigi. (In Japanese)

Noda, W. (2011). Japanese Elementary School Students' Luency of Academic Skills: Behavior Analytic Approach to Academic Instruction. Unpublished Doctoral Dissertation, Kwansei Gakuin University. (In Japanese)

Noda, W., \& Tanaka-Matsumi, J. (2014). Application of a Three-Tiered Instruction Model for Japanese Second-Grade Students to Improve Accuracy and Fluency of Multiplication Facts. Journal of Special Education Research, 2, 71-79.

https://doi.org/10.6033/specialeducation.2.71

Parker, R. L., Vannest, K. J., Davis, J. L., \& Sauber, S. B. (2011). Combining Nonoverlap and Trend for Single-Case Research: Tau-U. Behavior Therapy, 42, 284-299.

https://doi.org/10.1016/j.beth.2010.08.006

Rhymer, K. N., Skinner, C. H., Henington, C., D'Reaux, R. A., \& Sims, S. (1998). Effects of Explicit Timing on Mathematics Problem Completion Rates in African-American Third-Grade Elementary Students. Journal of Applied Behavior Analysis, 31, 673-677. https://doi.org/10.1901/jaba.1998.31-673

Skinner, C. H., Belfiore, P. J., \& Pierce, N. (1992). Cover, Copy and Compare: Increasing Geography Accuracy in Students with Behavior Disorders. School Psychology Review, 21,73-81.

Skinner, C. H., McLaughlin, T. E., \& Logan, P. L. (1997). Cover, Copy, and Compare: A Self-managed Academic Intervention Effective Across Skills, Students, and Settings. Journal of Behavioral Education, 7, 295-306. https://doi.org/10.1023/A:1022823522040

Skinner, C. H., Turco, T. L., Beatty, K. L., \& Rasavage, C. (1989). Cover, Copy, Compare: An Intervention for Increasing Multiplication Performance. School Psychology Review, $18,212-220$.

Stein, M., Kinder, D., Silbert, J., \& Carnine, D. W. (2006). Designing Effective Mathemat- 
ics Instruction: A Direct Instruction Approach (4th ed.). Upper Saddle River, NJ: Pearson Education Inc.

Tatsuno, T., Ishida, T., Hattori, T., Moriyama, T., Tanaka, H., Nakata, T. et al. (2009). Kyo-ken shikihyojungakuryokukensa NRT. [Kyoken-shiki Norm Referenced Standardized Achievement Test]. Tokyo: Toshobunka. (In Japanese)

Van Houten, R., \& Thompson, C. (1976). The Effects of Explicit Timing on Math Performance. Journal of Applied Behavior Analysis, 9, 227-230.

https://doi.org/10.1901/jaba.1976.9-227 